\title{
STRUCTURE AND REPRESENTATION OF NONASSOCIATIVE ALGEBRAS ${ }^{1}$
}

\author{
R. D. SCHAFER
}

1. Introduction. By a nonassociative algebra is meant a vector space which is equipped with a bilinear multiplication. If the multiplication is associative, we have the familiar notion of an associative algebra. Also Lie algebras are an essential tool for the study of Lie groups, as is well known. However, we shall not be particularly concerned with associative or Lie algebras today, except as models of what well-behaved nonassociative algebras should be.

The study of algebras which are not associative is not a recent development. The 8-dimensional algebra of Cayley numbers was known as early as $1845[26] .{ }^{2}$ However, it is within the last fifteen years that the study has received its greatest impetus. With a few notable exceptions - and always excepting Lie algebras of course-there were only isolated results before that time. By now a pattern is emerging, for certain finite-dimensional algebras at least, and this paper is an exposition of some of the principal results achieved recently in the structure and representation of finite-dimensional nonassociative algebras.

A. A. Albert has been the prime mover in this study. The depth and scope of the results, at least for one class of nonassociative algebras, may be judged by the fact that $\mathrm{N}$. Jacobson will give the Colloquium lectures at the Summer Meeting of the Society on the topic of Jordan algebras. Except for this reference to these two men, I shall not attempt to identify the authors of any theorems in this talk. The bibliography of the published paper will speak for itself.

2. The associative and Lie theories as models. Let $F$ be an arbitrary field and $A$ be a finite-dimensional associative algebra over $F$. It is well known that there is an ideal $N$, called the radical of $A$, which is the unique maximal nilideal of $A$ (that is, the maximal ideal consisting entirely of nilpotent elements). Furthermore, $N$ is nilpotent in the sense that there is an integer $t$ with the property that any product $z_{1} z_{2} \cdots z_{t}$ of $t$ elements from $N$ is zero; hence $N$ is also the

An address delivered before the Brooklyn meeting of the Society on April 15, 1955, by invitation of the Committee to Select Hour Speakers for Eastern Sectional Meetings; received by the editors May 31, 1955.

1 This paper was supported in part by a grant from the National Science Foundation.

${ }^{2}$ Numbers in brackers refer to the bibliography at the end of the paper. 
unique maximal nilpotent ideal of $A$. Modulo this radical the algebra is semisimple; that is, the difference algebra $A / N$ has radical equal to zero. Moreover, any semisimple associative algebra is uniquely expressible as a direct sum $S_{1} \oplus \cdots \oplus S_{r}$ of simple two-sided ideals (where an algebra is simple provided it has no proper ideals and is not a 1-dimensional algebra in which all products are zero). Any simple associative algebra $S$ is the Kronecker product $F_{s} \times D$ (over $F$ ) of the total matric algebra $F_{s}$ of dimension $s^{2}$ and a division algebra $D$ over $F$, where $s$ is unique and $D$ is uniquely determined up to isomorphism. Hence (up to a determination of all division algebras $D$ over $F$ ) the structure of any semisimple associative algebra over $F$ is known.

Let $A / N$ be separable (that is, the center of each simple component is a separable field over $F$; this would always be the case if $F$ were of characteristic 0). Then $A$ has a Wedderburn decomposition $A=S+N$ where $S$ is a subalgebra of $A$ isomorphic to $A / N$ and $S+N$ is a vector space direct sum. This decomposition is unique up to an inner automorphism of $A$ in the following strong sense. Let $z$ be any nilpotent element in $A$. Then, even though there is no identity element 1 in $A$, the meaning of $(1-z) a(1-z)^{-1}$ is clear, and the mapping $G_{z}$ : $a \rightarrow(1-z) a(1-z)^{-1}$ is an inner automorphism of $A$. Suppose that $A$ has Wedderburn decompositions $A=S+N=S_{1}+N$. Then there is an element $z$ of the radical $N$ such that the corresponding inner automorphism $G_{z}$ maps $S_{1}$ onto $S$ (and of course leaves $N$ invariant) [73].

This of course has been but the briefest of sketches of the associative structure theory, and omits many important features. It is astonishing, however, how closely the structure of Lie algebras of characteristic 0 parallels the associative theory up to this point.

Let $F$ be a field of characteristic 0 and $A$ be a finite-dimensional Lie algebra over $F$. Then the radical $N$ of $A$ is not the maximal nilideal (since $A$ itself is a nilalgebra, the square of every element being zero), nor is it in general the maximal nilpotent ideal of $A$. It is an ideal between these two. Define $B^{(1)}=B, B^{(i+1)}=\left(B^{(i)}\right)^{2}$. Then $B$ is solvable in case there is an integer $k$ such that $B^{(k)}=0$, and the radical $N$ of $A$ is the unique maximal solvable ideal of $A$. With this definition of radical, the difference algebra $A / N$ is semisimple and is uniquely expressible as a direct sum of simple two-sided ideals. If $F$ is algebraically closed, the classification of simple Lie algebras into four great classes and five exceptional algebras is well known. This leads to a determination of the simple Lie algebras over arbitrary $F$ of characteristic 0 which by now is almost complete, and in this sense we can say that all semisimple Lie algebras over $F$ are known. 
Since $F$ is of characteristic 0 , there is no question of separability involved. Any finite-dimensional Lie algebra $A$ over $F$ has a Levi decomposition $A=S+N$ where $S$ is a subalgebra isomorphic to $A / N$ and $S+N$ is a vector space direct sum. Moreover, we have a similar strict conjugacy of semisimple components in such decompositions. Let $z$ be any element of the maximal nilpotent ideal $N_{0}$ of $A\left(N_{0} \subseteq N\right)$, and let $D_{z}=$ ad $z$ (the right multiplication of $A$ determined by $z$ ) be the nilpotent inner derivation of $A$ corresponding to $z$. Then $G_{z}=\exp D_{z}$ is an inner automorphism of $A$. If $A=S+N=S_{1}+N$, then there is an element $z$ in $N_{0}$ such that $G_{z}$ maps $S_{1}$ onto $S$ [37]. ${ }^{3}$

Lest we be too taken up with the similarities between these two theories, we should perhaps recall one of the important tools for the study of associative algebras; namely, the Peirce decomposition relative to an idempotent. Let $e$ be an idempotent $\left(e^{2}=e \neq 0\right)$ in an associative algebra $A$ over an arbitrary field $F$. Then $A$ may be written as the vector space direct sum $A=A_{11}+A_{10}+A_{01}+A_{00}$ of spaces $A_{i j}$ which consist of those elements $a_{i j}$ in $A$ satisfying $e a_{i j}=i a_{i j}, a_{i j} e=j a_{i j}$ $(i, j=0,1)$. The properties of this decomposition are essential to the proofs of the associative theorems I have mentioned. On the other hand there are clearly no idempotents in Lie algebras, and the proofs of the parallel theorems rely on other methods, notably on a trace argument which breaks down for fields of characteristic $p>0$.

3. Arbitrary nonassociative algebras. Turning now to arbitrary nonassociative algebras, I should mention first that the concepts of subalgebra, ideal, homomorphism, isomorphism, simple algebra, difference algebra, and direct sum do not involve associativity in any way. If a nonassociative algebra can be written as the direct sum $S_{1} \oplus \cdots \oplus S_{r}$ of simple two-sided ideals $S_{i}$, it is easy to see that the simple summands in such a decomposition are uniquely determined. If we hope to use the associative and Lie theories as models at all, then we are forced to define a finite-dimensional nonassociative algebra to be semisimple in case it is a direct sum of simple two-sided ideals. (For some purposes it may be desirable to restrict the notion of simplicity, but that is a refinement which I can take up later.) The characteristic property of the radical then is not that it is the maximal nil-, solvable, or nilpotent ideal, but that it is the minimal ideal $N$ such that $A / N$ is zero or a direct sum of simple two-sided ideals [4]. Thus any nonassociative algebra $A$ has a radical $N$, and it is easy to see that the difference algebra $A / N$ is semisimple in the original sense that its radical is zero. Also $A / N$ is uniquely expressible

${ }^{3}$ It follows from the Campbell-Hausdorff formula that, if $G=G_{z_{1}} G_{z_{2}} \cdots G_{z_{h}}$ for $z_{i} \in N_{0}$, then there exists $z \in N_{0}$ such that $G=G_{z}$. 
as a direct sum of simple two-sided ideals.

Guided by the associative and Lie theories, one is then led to ask: what is the nature of the radical $N$, what are the simple algebras, what is the nature of $A$ as an extension of $N$ ? For an arbitrary algebra $A$, the answer is that anything can happen.

It is possible to construct a nonassociative algebra with almost any undesirable property. Most of these constructions are based on the use of a multiplication table. Let $u_{1}, \cdots, u_{n}$ be a basis for $A$ as an $n$-dimensional vector space over $F$. Then $u_{i} u_{j}=\sum_{k} \gamma_{i j k} u_{k}$ for uniquely determined elements $\gamma_{i j k}$ in $F$. Conversely, any $n^{2}$ equations of this type (that is, with $n^{3}$ multiplication constants $\gamma_{i j k}$ arbitrary in $F$ ) determine a nonassociative algebra of dimension $n$ over $F$, since the definition of multiplication is extended to products of arbitrary pairs in $A$ by bilinearity.

Let $A$ be a 2-dimensional algebra ${ }^{4}$ with basis $u, v$ and with multiplication table $u^{2}=u, u v=v^{2}=v, v u=0$. The 1 -dimensional ideal $N$ with basis $v$ is the only proper ideal of $A$. Since $A$ cannot be the direct sum of two proper ideals, $A$ is not semisimple. Then, since $A / N=F \bar{u} \cong F$ is semisimple, $N$ is the radical of $A$. But $v$ is idempotent, so $N=F v \cong F$ is a field. This is the extreme of being unlike a radical (in the nil-, solvable, or nilpotent sense).

Again let $A$ be a 2-dimensional algebra with basis $u, v$ and this time with multiplication table $u^{2}=u+v, u v=v, v u=v^{2}=0$. Again $N$ with basis $v$ is the only proper ideal of $A$ and, since $\bar{u}^{2}=\bar{u}, A / N=F \tilde{u} \cong F, N$ is the radical of $A$. Since $N$ is a zero algebra, this radical is wellbehaved. However, there cannot be any Wedderburn decomposition for $A$. For there is no idempotent $e$ in $A$, as there would have to be for $A$ to contain a subalgebra $S \cong A / N \cong F$.

The literature contains other unpleasant examples, some not nearly as easily constructed as these. They serve to put limits on our expectations for a general theory, and focus our attention on classes of algebras for which associative algebras and Lie algebras of characteristic 0 serve as reasonable models. They also increase our appreciation of those techniques which are applicable to arbitrary nonassociative algebras.

Let $a$ be an element in a nonassociative algebra $A$ over $F$, and $R_{a}$ be the right multiplication of $A$ determined by $a$; that is, $R_{a}$ is the mapping $x \rightarrow x a=x R_{a}$ for every $x$ in $A$. Similarly for the left multiplication $L_{a}: x \rightarrow a x=x L_{a}$. The right and left multiplications are elements of $F_{n}$, the total matric algebra of all linear transformations on the vector space $A$ of dimension $n$. We denote by $E$ the associative subalgebra

${ }^{4}$ This example is a modification of the 3-dimensional example in [4]. 
of $F_{n}$ generated by the right and left multiplications of $A$. The structural properties of $A$ are reflected in those of its associative multiplication algebra $E[41 ; 3]$. If $A$ is semisimple, then so is $E$. If $A$ is simple, then so is $E$, and $E$ is indeed a total matrix algebra over its center. The radical of $A$ may be characterized in terms of the radical of $E[4 ; 57]$.

The center of a nonassociative algebra is the set of elements $c$ which not only commute with every element of $A$ but associate with every pair of elements $a, b$ in $A$. If $A$ is simple, the center of $A$ is either 0 or a field. In the latter case $A$ may be regarded as an algebra over its center, and as such is central simple (that is, remains simple under scalar extension of the ground field). In any event the center of $E$ is a field, the multiplication center of $A$, and $A$ is a central simple algebra over its multiplication center $[41 ; 3 ; 44]$. A nonassociative algebra is separable (that is, is semisimple over any scalar extension of the ground field) if and only if the multiplication centers of its simple summands are separable fields.

Solvability of an algebra $\left(B^{(k)}=0\right)$ is a nonassociative notion. It turns out that the radical of an algebra always contains the maximal solvable ideal [5] (although we have seen an example showing that it can contain much more). Nilpotence of an algebra can be turned into a nonassociative notion by demanding that there is an integer $t$ such that any product $z_{1} z_{2} \cdots z_{t}$ of $t$ elements, no matter how associated, is zero. Clearly any nilpotent algebra is solvable. Moreover, any ideal $N_{0}$ of $A$ is nilpotent if and only if the subalgebra of $E$ generated by the right and left multiplications corresponding to elements of $N_{0}$ is a nilpotent associative algebra $[5 ; 33]$.

There are also relationships between arbitrary nonassociative algebras and Lie algebras. A derivation $D$ of a nonassociative algebra $A$ is a linear transformation on $A$ having the property of differentiation: $(x y) D=x(y D)+(x D) y$. The set of all derivations of $A$ is a Lie algebra (with product the ordinary commutator $\left[D, D^{\prime}\right]=D D^{\prime}-D^{\prime} D$ ), and this is called the derivation algebra of $A$ [42]. The Lie enveloping algebra of the right and left multiplications of $A$ (that is, the algebra generated by addition and commutation, rather than addition and multiplication) is called the Lie multiplication algebra $L$ of $A$ [88; 47]. If $A$ is semisimple of characteristic 0 , and if $A$ has an identity element 1 , then every derivation $D$ of $A$ is inner in the sense that $D$ is in $L$ [47]. For associative and Lie algebras inner derivations have the familiar forms $D=R_{x}-L_{x}$ and $D=R_{x}(=\operatorname{ad} x)$ respectively.

4. Alternative and Jordan algebras. Let us turn now to consideration of two classes of algebras whose behavior, according to the norms 
we have set, is excellent. These are the well-known alternative and Jordan algebras. It turns out that they are intimately interrelated.

An algebra $A$ is called alternative in case the identities $a x^{2}=(a x) x$, $x^{2} a=x(x a)$ are satisfied in $A$. Clearly all associative algebras are alternative. It turns out that the only simple alternative algebras which are not associative are 8-dimensional algebras over their centers which are generalizations of the original algebra of Cayley numbers $[109 ; 110 ; 83]$. Hence all semisimple alternative algebras are known.

Any alternative algebra is power-associative (that is, if $x^{1}=x$, and $x^{i+1}=x^{i} x$, we have $x^{j} x^{k}=x^{j+k}$ so that all products of $m$ factors, each equal to $x$, are equal and we can write this common element as $x^{m}$ ). Hence nilpotence of an element is unambiguously defined. The radical $N$ of $A$ is the maximal nilideal, and $N$ is nilpotent $[112 ; 87]$. If $A / N$ is separable, then $A$ has a Wedderburn decomposition $A=S+N$ where $S \cong A / N$ [87]. All of this is for an arbitrary field $F$, and the proofs rely heavily on the fact that there is a Peirce decomposition of $A$ whose properties are identical with those of the special case of associative algebras $[109 ; 112]$.

If $F$ is of characteristic 0 , we can prove the strict conjugacy of semisimple summands in Wedderburn decompositions. If $F$ is of characteristic $p$, the question is still open, and the solution depends on arriving at a suitable form for the inner automorphisms of $A$. The derivations are more tractable [88]. If $A=S+N=S_{1}+N$ is of characteristic 0 , then there is a nilpotent derivation $D$ of $A$ in the radical of $E$ such that the automorphism $G=\exp D$ of $A$ maps $S_{1}$ onto $S$ [90]. Moreover, any alternative algebra of characteristic 0 is semisimple if and only if its derivation algebra is semisimple or $0[90] .{ }^{5}$

Jordan algebras are also defined by identities: $x a=a x,\left(x^{2} a\right) x$ $=x^{2}(a x)$. Since the second of these identities is a weak form of associativity, any commutative associative algebra is a Jordan algebra. Let us assume henceforth that the characteristic of $F$ is not two. The most easily realizable Jordan algebras are those obtained from associative algebras as follows. Let $B$ be any associative algebra with multiplication denoted by $x \cdot y$. Consider a new algebra $B^{+}$which is the same vector space as $B$, but in which multiplication is defined by $x y=(x \cdot y+y \cdot x) / 2 . B^{+}$is clearly commutative, and it is easy to verify that the Jordan identity is also satisfied. Any nonassociative algebra $A$ over $F$ which is isomorphic to a subalgebra of some $B^{+}$is called a special Jordan algebra. The special Jordan algebras are not defined

5 This generalizes the associative theorem in [38]. The analogue for Jordan algebras is proved in [89]. 
by identities, since a homomorphic image of a special Jordan algebra need not be special [28].

Any Jordan algebra (of characteristic $\neq 2$ ) is power-associative. The radical $N$ is the maximal nilideal, and $N$ is nilpotent [6]. Every simple Jordan algebra has an identity element 1 , and is therefore central simple over its center. With a possible exception which can occur only if the characteristic is $p>0$, all central simple Jordan algebras are known. The degree of a central simple algebra is the unique number $q$ of the pairwise orthogonal primitive idempotents $e_{i}$ in $1=e_{1}+\cdots+e_{q}$ over any algebraically closed extension $\Omega$ of $F$. It is an open question whether any central simple Jordan algebras of degree one and dimension greater than one exist. If they do, they are of characteristic $p>0$, and they are exceptional in the sense that they are not special Jordan algebras [13]. With this possible exception, there are four great classes of central simple Jordan algebras (all of which are special) - algebras of degree two defined by quadratic forms and three classes of algebras of self-adjoint elements in certain involutorial associative algebras $[61 ; 5 ; 6 ; 62 ; 45 ; 40 ; 13]$-and one class of exceptional algebras which are 27 -dimensional generalizations of the algebra of 3 -rowed hermitian matrices with elements which are Cayley numbers $[1 ; 85 ; 12 ; 13]$.

If $A / N$ is a known separable Jordan algebra, ${ }^{6}$ then $A$ has a Wedderburn decomposition $A=S+N$ where $S \cong A / N[80 ; 20]$. If $A=S+N$ $=S_{1}+N$ is of characteristic 0 , then there is a nilpotent derivation $D$ of $A$ in the radical of $E$ such that the automorphism $G=\exp D$ of $A$ maps $S_{1}$ onto $S$ [49].

The proofs of these theorems depend on the decomposition of a Jordan algebra relative to an idempotent $[61 ; 6]$. This decomposition is quite evident in case $A=B^{+}$, for $e$ is idempotent in $B^{+}$if and only if $e$ is idempotent in $B$. Then $B=B_{11}+B_{10}+B_{01}+B_{00}$ and $b_{i j}$ in $B_{i j}$ give $b_{i j} e=\left(b_{i j} \cdot e+e \cdot b_{i j}\right) / 2=(i+j) b_{i j} / 2$ and $A=A_{1}+A_{1 / 2}+A_{0}$ where $A_{1}=B_{11}, A_{1 / 2}=B_{10}+B_{01}, A_{0}=B_{00}$. The same decomposition $A=A_{1}$ $+A_{1 / 2}+A_{0}$, with $a_{i} e=i a_{i}$ for $a_{i}$ in $A_{i}$, holds for any idempotent $e$ in a general Jordan algebra $A$, and the properties of this decomposition are fundamental in the structure theory.

5. Power-associative algebras. All of the algebras which we have considered have been power-associative (that is, each single element generates an associative subalgebra). It is natural to try to see how far the results can be extended to power-associative algebras $[7 ; 8]$. We are always assuming now that the characteristic of $F$ is not two.

${ }^{6}$ The proof in [80] is valid for $F$ of characteristic $\neq 2$ in case each simple summand of (the separable algebra) $A / N$ is a known simple Jordan algebra. 
Let us begin with commutative power-associative algebras. Relative to any idempotent $e$ there is a decomposition $A=A_{1}+A_{1 / 2}+A_{0}$ which enjoys most of the properties of this decomposition for Jordan algebras $[8 ; 13]$. We modify the definition of a simple algebra, demanding that the algebra not be a nilalgebra as well as not having any proper ideals. The radical of $A$ is then the minimal ideal $N$ such that $A / N$ is a direct sum of such simple algebras. We can either further restrict the characteristic of $F$ to be $\neq 3,5$, or assume that $A$ is strictly power-associative in the sense that $A_{K}$ is power-associative for every scalar extension $K$ of $F$. Then $N$ is the maximal nilideal of $A$. Every simple algebra has an identity element 1 . Moreover, the only simple algebras of degree greater than 2 are the known simple Jordan algebras $[13 ; 15 ; 69]$. An interesting feature of the technique used in obtaining these results is that the result on simple algebras is used to prove that the maximal nilideal is the radical.

If $F$ is of characteristic $p>5$, there do exist examples of simple commutative power-associative algebras of degree 2 which are not Jordan algebras [68]. However, it is an open question ${ }^{7}$ whether such examples exist for $F$ of characteristic 0 . The strongest theorem for algebras of degree 2 is that $A$ is a Jordan algebra if and only if $A_{i} A_{1 / 2} \subseteq A_{1 / 2}(i=0,1)$ for every idempotent $e$ in $A$ [18]. If $F$ is of characteristic 0 , then any simple commutative power-associative algebra of degree 1 is 1-dimensional over its center [13].

Whether or not the new definition of simplicity is an actual restriction is not known. That is, whether or not commutative powerassociative nilalgebras without proper ideals exist (except of course for the zero algebra of dimension 1) is still an open problem. Whether or not the radical is nilpotent (or solvable) is a related question. To my knowledge nothing is known about Wedderburn decompositions for commutative power-associative algebras.

The situation with respect to noncommutative algebras is rather chaotic. However, pleasing results are known for some classes of algebras. The most effective tool is the passage from $A$ to the attached commutative algebra $A^{+}$in which multiplication is defined by $x \mathrm{O} y=(x y+y x) / 2$ where $x y$ is the product in $A$ [8]. Whether or not the method is successful depends on how much is lost in transition from $A$ to $A^{+}$. For example, if $A$ is anticommutative $(a x=-x a)$, then everything is lost because $x \mathrm{O} y=0$.

Noncommutative Jordan algebras [94] are defined by the Jordan

${ }^{7}$ L. A. Kokoris has subsequently announced (April 22, 1955) that every simple commutative power-associative algebra of degree 2 and characteristic 0 is a Jordan algebra. 
identity $\left(x^{2} a\right) x=x^{2}(a x)$ and an identity which is a consequence of this if $A$ has an identity element: $(x a) x=x(a x)$. The latter is called the flexible law [8], and is satisfied in any commutative (or anticommutative) algebra. Noncommutative Jordan algebras are powerassociative, and they include Jordan, alternative, and-triviallyLie algebras. They also include the quasiassociative algebras $A$ (over some scalar extension $K$ of $F$ there is an associative algebra $B$ and a scalar $\lambda \neq 1 / 2$ in $K$ such that $A_{K} \cong B(\lambda)$, the algebra which is the same vector space as $B$ but in which multiplication is defined by $x y=\lambda x \cdot y$ $+(1-\lambda) y \cdot x$ where $x \cdot y$ is the product in $B)$ [8]. The attached algebra $A^{+}$of any noncommutative Jordan algebra $A$ is a (commutative) Jordan algebra. If the characteristic of $F$ is 0 , then $A$ is traceadmissible according to the following definition. There is a linear function $\tau(x)$ on $A$ (namely, the trace of the right multiplication of $A^{+}$corresponding to $\left.x\right)$ with the following properties: $\tau(x y)=\tau(y x)$, $\tau((x y) z)=\tau(x(y z)), \tau(e) \neq 0$ for any idempotent $e$ of $A, \tau(x)=0$ if $x$ is nilpotent. But for any trace-admissible algebra, if the definition of simplicity includes not being a nilalgebra, it is known [10] that the radical is the maximal nilideal and is the set of all $z$ in $A$ satisfying $\tau(x z)=0$ for every $x$. The radical is not nilpotent, or even solvable, since every Lie algebra is its own radical by this definition (in which the simple algebras are taken to be not nilalgebras). The simple algebras are the simple (commutative) Jordan algebras, the simple flexible algebras of degree two, and the simple quasiassociative algebras. One can construct an example ${ }^{8}$ to show that noncommutative Jordan algebras do not in general have Wedderburn decompositions (even in case $N^{2}=0$ ).

Right alternative algebras are defined by the identity $a x^{2}=(a x) x$. If $A$ is right alternative, then $A^{+}$is a special Jordan algebra. By a modification of the trace-admissibility technique, it can be shown that for $F$ of arbitrary characteristic $\neq 2$ the radical of $A$ is its maxi-

\footnotetext{
${ }^{8}$ Let $A$ be the 5-dimensional noncommutative Jordan algebra with basis $e_{11}, e_{12}$, $e_{21}, e_{22}, z$ and multiplication table $e_{11}^{2}=e_{12} e_{21}=e_{11}, e_{11} e_{12}=e_{12} e_{22}=e_{12}, e_{21} e_{12}=e_{22}^{2}=e_{22}$, $e_{11} e_{21}=e_{21} e_{22}=e_{22} z=z e_{11}=z, e_{21} e_{11}=e_{22} e_{21}=e_{21}-z$, all other products zero. The radical $N$ is spanned by $z, N^{2}=0$, and $A / N \cong F_{2}$ with basis $\bar{e}_{11}, \bar{e}_{12}, \bar{e}_{21}, \bar{e}_{22}$. Suppose there were a subalgebra $S \cong A / N$. Then $S$ would have a usual matrix basis $g_{11}, g_{12}, g_{21}, g_{22}$, and there would be an automorphism $H$ of $A / N$ such that $\bar{g}_{i j}=\bar{e}_{i j} H$. But any automorphism of $A / N$ is inner, as there is a nonsingular element $\bar{y}=\alpha \bar{e}_{11}+\beta \bar{e}_{12}+\gamma \bar{e}_{21}+\delta \bar{e}_{22}$ $(\Delta=\alpha \delta-\beta \gamma \neq 0)$ in $A / N$ such that $\bar{g}_{i j}=\bar{y} \bar{e}_{i j} \bar{y}^{-1}$. It follows that $g_{11}=\Delta^{-1}\left(\alpha \delta e_{11}-\alpha \beta e_{12}\right.$ $\left.+\gamma \delta e_{21}-\beta \gamma e_{22}\right)+\epsilon z, \quad g_{12}=\Delta^{-1}\left(-\alpha \gamma e_{11}+\alpha^{2} e_{12}-\gamma^{2} e_{21}+\alpha \gamma e_{22}\right)+\kappa z, \quad g_{21}=\Delta^{-1}\left(\beta \delta e_{11}-\beta^{2} e_{12}\right.$ $\left.+\delta^{2} e_{21}-\beta \delta e_{22}\right)+\lambda z, g_{22}=\Delta^{-1}\left(-\beta \gamma e_{11}+\alpha \beta e_{12}-\gamma \delta e_{21}+\alpha \delta e_{22}\right)+\mu z$. Equating coefficients of $z$ in $g_{11} g_{12}=g_{12}, g_{22} g_{12}=g_{11} g_{21}=0, g_{22} g_{21}=g_{21}, g_{21} g_{12}=g_{22}$ yields equations in $\alpha, \beta, \cdots, \mu$ which force $\Delta=0$, a contradiction.
} 
mal nilideal, and that every semisimple right alternative algebra is actually alternative $[9 ; 19]$.

Every finite strictly power-associative division ring of characteristic $\neq 2$ is a finite field [17]. This generalizes Wedderburn's celebrated theorem on finite associative division rings. The proof appeals directly to the structure theory for the attached commutative powerassociative algebra $A^{+}$over the prime field $F$.

If $A$ is a simple power-associative algebra, then there is no reason why the commutative power-associative algebra $A^{+}$need be simple. If, however, $A^{+}$is simple, then it is clear that $A$ must be simple. If $A^{+}$is central simple of degree greater than 2 , then $A^{+}$is a known Jordan algebra and (at least over a scalar extension $K$ of $F$ ) the multiplication in $A$ can be given by an explicit construction $[14 ; 15]$, the details of which I do not have time to relate.

6. Modules and representations. A very valuable technique which has been introduced in the last few years is the use of modules for nonassociative algebras (or bimodules, since the concept is a generalization of the familiar two-sided module for associative algebras). An equivalent notion is that of a representation (or birepresentation).

Let $C$ be a class of nonassociative algebras over $F$. If $A$ is in $C$, and if $M$ is a vector space over $F$, let $m a$ and $a m$ in $M$ be two bilinear compositions for $a$ in $A, m$ in $M$. Then the direct sum $A+M$ of the vector spaces $A$ and $M$ is turned into a nonassociative algebra over $F$ (the semidirect sum, or split null extension) by defining multiplication in $A+M$ by $\left(a_{1}+m_{1}\right)\left(a_{2}+m_{2}\right)=a_{1} a_{2}+\left(m_{1} a_{2}+a_{1} m_{2}\right)$. If $A+M$ is in $C$, then $M$ is called a module for $A$ in $C$. For example, if $C$ is the class of all nonassociative algebras over $F$, then no conditions other than the originally assumed bilinearity are imposed on the compositions $a m$ and $m a$. If $C$ is the class of all associative algebras, then the compositions in $M$ must satisfy $(m a) b=m(a b),(a m) b=a(m b)$, (ab) $m=a(b m)$ for $a, b$ in $A, m$ in $M$; that is, $M$ is a two-sided $A$-module for the associative algebra $A$ [38]. If $C$ is any class of nonassociative algebras defined by multilinear identities $I_{i}\left(a_{1}, a_{2}, \cdots, a_{n_{i}}\right)=0$, then $M$ is a module for $A$ in $C$ in case all of the identities, obtained by replacing any single $a_{j}$ in $A$ in the identities $I_{i}\left(a_{1}, a_{2}, \cdots, a_{n_{i}}\right)=0$ by $m$ in $M$, are satisfied [31].

If $M$ is a module for $A$, then the mappings $S_{a}: m \rightarrow m a$ and $T_{a}: m \rightarrow a m$ are linear transformations on $M$, and $a \rightarrow S_{a}, a \rightarrow T_{a}$ are linear mappings of $A$ into the space of all linear transformations on $M$. A pair $(S, T)$ of linear mappings $a \rightarrow S_{a}, a \rightarrow T_{a}$ of $A$ into the space of all linear transformations on a vector space $M$ is called a representation of $A$ in $C$ in case $M$, equipped with the compositions $m a=m S_{a}$, 
$a m=T m_{a}$, is a module for $A$ in $C$. Any algebra $A$ in a class $C$ defined by identities has the regular representation $(R, L)$ where $M$ is $A$ itself, and $R_{a}$ and $L_{a}$ are right and left multiplications in $A$. An important simplification is possible if $C$ contains only commutative (or anticommutative) algebras. For then $m a=a m$ (or $m a=-a m$ ) implies $S_{a}=T_{a}$ (or $S_{a}=-T_{a}$ ), so that effectively only one mapping $S$ : $a \rightarrow S_{a}$ is involved instead of the pair $(S, T)$. This simplification can be made for Jordan and Lie algebras (for Lie algebras the notion of representation is the usual one).

The defining identities for alternative and Jordan algebras may be linearized. ${ }^{9}$ Modules and representations for alternative and Jordan algebras ${ }^{10}$ are then easily defined, and these have already proved to be very effective in the study of subalgebras of these algebras $[49 ; 90$; 53 ].

The analogues for alternative and Jordan algebras of the first Whitehead lemma $[38 ; 49 ; 90]$ depend on representation theory, as do the proofs of the theorems concerning the strict conjugacy of semisimple components in Wedderburn decompositions which I have already mentioned. Representations have also been used to prove that, if $B$ is an alternative algebra with an identity 1 and if $A$ is a Cayley subalgebra containing 1 , then $B$ is the Kronecker product $B=A \times Z$ (over $F$ ) where $Z$ is the center of $B$. Similarly, if $B$ is a Jordan algebra with identity 1 and if $A$ is a known (27-dimensional) exceptional simple subalgebra containing 1 , then $B$ is also the Kronecker product $B=A \times Z[54]$.

Just as the multiplication algebras $E$ and $L$ (which I have already mentioned) are generated by the elements $R_{a}$ and $L_{a}$ of the regular representation, there are associative (and Lie) enveloping algebras for any representation $(S, T)$. For alternative and Jordan algebras $A$ one can define a universal associative algebra $U$ for the representations, universal in the sense that any representation of $A$ is obtained from a representation (in the usual sense of homomorphism) of the associative algebra $U$ [53]. Furthermore, $U$ is finite-dimensional for finite-dimensional alternative and Jordan algebras $A[49 ; 90]$.

If $A$ is a separable alternative algebra or a known separable Jordan algebra, then every module for $A$ is completely reducible. The irreducible modules for these algebras have been determined [53].

\footnotetext{
9 The multilinear identities obtained are equivalent to the original ones if $F$ is of characteristic $\neq 2$ for alternative algebras and of characteristic $\neq 2,3$ for Jordan algebras.

${ }^{10}$ Included among the representations of Jordan algebras are the special representations defined by $S_{a b}=S_{a} S_{b}+S_{b} S_{a}[22 ; 40]$.
} 
There are intimations of cohomology in some of these results on alternative and Jordan algebras $[49 ; 90 ; 91 ; 53]$. One can hope for the development of a cohomology theory, not only for these algebras, but one for general nonassociative algebras which will provide a measure of the deviation of nonassociative algebras from the norms of good behavior which are gradually appearing.

7. Conclusion. In this short space of an hour I have had to omit many topics. I have made no mention of rings or infinite-dimensional algebras $[102 ; 99 ; 16 ; 63 ; 24 ; 67 ; 55 ; 56 ; 100 ; 101 ; 78 ; 70 ; 11 ; 64]$. Moreover, I have not touched on the interesting relationships between nonassociative algebras and the exceptional simple Lie algebras $[43 ; 27 ; 35 ; 105]$. Nor has there been time to explain how some of these algebras arise in other branches of mathematics $[29 ; 75 ; 36$; $95 ; 98 ; 104 ; 103 ; 39]$ and in the formalism of quantum mechanics $[58 ; 59 ; 60]$ and genetics $[32 ; 33 ; 34 ; 86]$. I hope, however, that I have been able to give the nonspecialist some insight into the results and problems of a rich and active field of research.

\section{BIBLIOGRAPHY}

1. A. A. Albert, On a certain algebra of quantum mechanics, Ann. of Math. vol. 35 (1934) pp. 65-73.

2. - Quadratic forms permitting composition, Ann. of Math. vol. 43 (1942) pp. 161-177.

3. - Non-associative algebras, I, Ann. of Math. vol. 43 (1942) pp. 685-707; II, ibid. pp. 708-723.

4. - The radical of a non-associative algebra, Bull. Amer. Math. Soc. vol. 48 (1942) pp. 891-897.

5. - On Jordan algebras of linear transformations, Trans. Amer. Math. Soc. vol. 59 (1946) pp. 524-555.

6. - A structure theory for Jordan algebras, Ann. of Math. vol. 48 (1947) pp. 546-567.

7. - On the power-associativity of rings, Summa Brasiliensis Mathematicae vol. 2 (1948) pp. 21-32.

8. - Power-associative rings, Trans. Amer. Math. Soc. vol. 64 (1948) pp. 552-593.

9. - On right alternative algebras, Ann. of Math. vol. 50 (1949) pp. 318-328.

10. - A theory of trace-admissible algebras, Proc. Nat. Acad. Sci. U.S.A. vol. 35 (1949) pp. 317-322.

11. - Absolute-valued algebraic algebras, Bull. Amer. Math. Soc. vol. 55 (1949) pp. 763-768; A note of correction, Bull. Amer. Math. Soc. vol. 55 (1949) p. 1191.

12. - A note on the exceptional Jordan algebra, Proc. Nat. Acad. Sci. U.S.A. vol. 36 (1950) pp. 372-374.

13. - A theory of power-associative commutative algebras, Trans. Amer. Math. Soc. vol. 69 (1950) pp. 503-527.

14. - New simple power-associative algebras, Summa Brasiliensis Mathematicae vol. 2 (1951) pp. 183-194. 
15. - Power-associative algebras, Proceedings of the International Congress of Mathematicians, Cambridge, Mass., 1950, Providence, American Mathematical Society, 1952, vol. 2, pp. 2-32.

16. - On simple alternative rings, Canadian Journal of Mathematics vol. 4 (1952) pp. 129-135.

17. - On nonassociative division algebras, Trans. Amer. Math. Soc. vol. 72 (1952) pp. 296-309.

18. - On commutative power-associative algebras of degree two, Trans. Amer. Math. Soc. vol. 74 (1953) pp. 323-343.

19. - The structure of right alternative algebras, Ann. of Math. (2) vol. 59 (1954) pp. 408-417.

20. V. G. Askinuze, $A$ theorem on the splittability of J-algebras, Ukrain Mat. Zurnal. vol. 3 (1951) pp. 381-398 (Russian).

21. E. A. Behrens, Nichtassoziative Ringe, Math. Ann. vol. 127 (1954) pp. 441452.

22. G. Birkhoff and P. M. Whitman, Representations of Jordan and Lie algebras, Trans. Amer. Math. Soc. vol. 65 (1949) pp. 116-136.

23. R. H. Bruck, Some results in the theory of linear non-associative algebras, Trans. Amer. Math. Soc. vol. 56 (1944) pp. 141-199.

24. R. H. Bruck and E. Kleinfeld, The structure of alternative division rings, Proc. Amer. Math. Soc. vol. 2 (1951) pp. 878-890.

25. H. E. Campbell, An extension of the "principal theorem" of Wedderburn, Proc. Amer. Math. Soc. vol. 2 (1951) pp. 581-585.

26. A. Cayley, On Jacobi's elliptic functions, in reply to the Rev. Brice Bronwin; and on quaternions, Philosophical Magazine vol. 26 (1845) pp. 210-213.

27. C. Chevalley and R. D. Schafer, The exceptional simple Lie algebras $F_{4}$ and $E_{6}$, Proc. Nat. Acad. Sci. U.S.A. vol. 36 (1950) pp. 137-141.

28. P. M. Cohn, On homomorphic images of special Jordan algebras, Canadian Journal of Mathematics vol. 6 (1954) pp. 253-264.

29. L. E. Dickson, On quaternions and their generalization and the history of the eight square theorem, Ann. of Math. vol. 20 (1919) pp. 155-171.

30. R. Dubisch and S. Perlis, On the radical of a non-associative algebra, Amer. J. Math. vol. 70 (1948) pp. 540-546.

31. S. Eilenberg, Extensions of general algebras, Annales de la Société Polonaise de Mathématique vol. 21 (1948) pp. 125-134.

32. I. M. H. Etherington, Genetic algebras, Proceedings of the Royal Society of Edinburgh vol. 59 (1939) pp. 242-258.

33. —_, Special train algebras, Quart. J. Math. Oxford Ser. vol. 12 (1941) pp. 1-8.

34. - Non-associative algebra and the symbolism of genetics, Proceedings of the Royal Society of Edinburgh vol. 61 (1941) pp. 24-42.

35. H. Freudenthal, Oktaven, Ausnahmengruppen und Oktavengeometrie, Utrecht, 1951.

36. M. Hall, Projective planes, Trans. Amer. Math. Soc. vol. 54 (1943) pp. 229277. Correction to Projective planes, ibid. vol. 65 (1949) p. 474.

37. Harish-Chandra, On the radical of a Lie algebra, Proc. Amer. Math. Soc. vol. 1 (1950) pp. 14-17.

38. G. P. Hochschild, Semi-simple algebras and generalized derivations, Amer. J. Math. vol. 64 (1942) pp. 677-694.

39. H. Hopf, Ein topologischer Beitrag zur reellen Algebra, Comment. Math. Helv. vol. 13 (1941) pp. 219-239. 
40. F. D. Jacobson and N. Jacobson, Classification and representation of semisimple Jordan algebras, Trans. Amer. Math. Soc. vol. 65 (1949) pp. 141-169.

41. N. Jacobson, $A$ note on non-associative algebras, Duke Math. J. vol. 3 (1937) pp. 544-548.

42. —, Abstract derivation and Lie algebras, Trans. Amer. Math. Soc. vol. 42 (1937) pp. 206-224.

43. - Cayley numbers and normal simple Lie algebras of type $G$, Duke Math. J. vol. 5 (1939) pp. 775-783.

44. - Structure theory of simple rings without finiteness assumptions, Trans. Amer. Math. Soc. vol. 57 (1945) pp. 228-245. 326.

45. — - Isomorphisms of Jordan rings, Amer. J. Math. vol. 70 (1948) pp. 317-

46. - The center of a Jordan ring, Bull. Amer. Math. Soc. vol. 54 (1948) pp. $316-322$.

47. - Derivation algebras and multiplication algebras of semi-simple Jordan algebras, Ann. of Math. vol. 50 (1949) pp. 866-874. 170.

48. —_, Lie and Jordan triple systems, Amer. J. Math. vol. 71 (1949) pp. 149-

49. - General representation theory of Jordan algebras, Trans. Amer. Math. Soc. vol. 70 (1951) pp. 509-530.

50. - Representation theory for Jordan rings, Proceedings of the International Congress of Mathematicians, Cambridge, Mass., 1950, Providence, American Mathematical Society, 1952, vol. 2, pp. 37-43.

51. — Une generalisation du théorème d'Engel, C. R. Acad. Sci. Paris vol. 234 (1952) pp. 579-581.

52. - Operator commutativity in Jordan algebras, Proc. Amer. Math. Soc. vol. 3 (1952) pp. 973-976.

53. - Structure of alternative and Jordan bimodules, Osaka Math. J. vol. 6 (1954) pp. 1-71.

54. - A Kronecker factorization theorem for Cayley algebras and the exceptional simple Jordan algebra, Amer. J. Math. vol. 76 (1954) pp. 447-452.

55. N. Jacobson and C. E. Rickart, Jordan homomorphisms of rings, Trans. Amer. Math. Soc. vol. 69 (1950) pp. 479-502.

56. - Homomorphisms of Jordan rings of self-adjoint elements, Trans. Amer. Math. Soc. vol. 72 (1952) pp. 310-322.

57. W. E. Jenner, The radical of a non-associative ring, Proc. Amer. Math. Soc. vol. 1 (1950) pp. 348-351.

58. P. Jordan, Über eine Klasse nichtassoziativer hyperkomplexer Algebren, Nachr. Ges. Wiss. Göttingen, 1932, pp. 569-575.

59. - Ü̈ber Verallgemeinerungsmöglichkeiten des Formalismus der Quantenmechanik, Nachr. Ges. Wiss. Göttingen (1933) pp. 209-214.

60. — Ü̈ber die Multiplication quantenmechanischer Grössen, Zeitschrift für Physik vol. 80 (1933) pp. 285-291.

61. P. Jordan, J. von Neumann, and E. Wigner, On an algebraic generalization of the quantum mechanical formalism, Ann. of Math. vol. 35 (1934) pp. 29-64.

62. G. K. Kalisch, On special Jordan algebras, Trans. Amer. Math. Soc. vol. 61 (1947) pp. 482-494.

63. I. Kaplansky, Semisimple alternative rings, Portugaliae Mathematica vol. 10 (1951) pp. 37-50.

64. - Infinite-dimensional quadratic forms permitting composition, Proc. Amer. Math. Soc. vol. 4 (1953) pp. 956-960. 
65. E. Kleinfeld, Alternative division rings of characteristic 2, Proc. Nat. Acad. Sci. U.S.A. vol. 37 (1951) pp. 818-820. 944.

66. — , Right alternative rings, Proc. Amer. Math. Soc. vol. 4 (1953) pp. 939-

67. - Simple alternative rings, Ann. of Math. vol. 58 (1953) pp. 544-547.

68. L. A. Kokoris, Power-associative commutative algebras of degree two, Proc. Nat. Acad. Sci. U.S.A. vol. 38 (1952) pp. 534-537.

69. - New results on power-associative algebras, Trans. Amer. Math. Soc. vol. 77 (1954) pp. 363-373.

70. A. Kurosch, Non-associative free algebras and free products of algebras, Rec. Math. (Mat. Sbornik) N.S. vol. 20 (62) (1947) pp. 239-262 (Russian).

71. - The present status of the theory of rings and algebras, Uspehi Matematičeskih Nauk (N.S.) vol. 6, no. 2 (42) (1951) pp. 3-15 (Russian).

72. W. G. Lister, A structure theory of Lie triple systems, Trans. Amer. Math. Soc. vol. 72 (1952) pp. 217-242.

73. A. Malcev, On the representation of an algebra as a direct sum of the radical and a semisimple subalgebra, C. R. (Doklady) Acad. Sci. URSS vol. 36 (1942) pp. 42-45.

74. - On a representation of nonassociative rings, Uspehi Matematičeskih Nauk (N.S.) vol. 7, no. 1 (47) (1952) pp. 181-185 (Russian).

75. R. Moufang, Alternativkörper und der Satz vom vollständigen Vierseit $\left(D_{9}\right)$, Abh. Math. Sem. Hamburgischen Univ. vol. 9 (1933) pp. 207-222.

76. —_, Zur Struktur von Alternativkörpern, Math. Ann. vol. 110 (1935) pp. 416-430.

77. W. H. Mills, $A$ theorem on the representation theory of Jordan algebras, Pacific Journal of Mathematics vol. 1 (1951) pp. 255-264.

78. B. H. Neumann, Embedding non-associative rings in division rings, Proc. London Math. Soc. (3) vol. 1 (1951) pp. 241-256.

79. L. J. Paige, $A$ theorem on power-associative loop algebras, Proc. Amer. Math. Soc. vol. 6 (1955) pp. 279-280.

80. A. J. Penico, The Wedderburn principal theorem for Jordan algebras, Trans. Amer. Math. Soc. vol. 70 (1951) pp. 404-420.

81. C. M. Price, Jordan division algebras and the algebras $A(\lambda)$, Trans. Amer. Math. Soc. vol. 70 (1951) pp. 291-300.

82. R. L. San Soucie, Right alternative division rings of characteristic 2, Proc. Amer. Math. Soc. vol. 6 (1955) pp. 291-296.

83. R. D. Schafer, Alternative algebras over an arbitrary field, Bull. Amer. Math. Soc. vol. 49 (1943) pp. 549-555.

84. - Concerning automorphisms of non-associative algebras, Bull. Amer. Math. Soc. vol. 53 (1947) pp. 573-583.

85. — The exceptional simple Jordan algebras, Amer. J. Math. vol. 70 (1948) pp. 82-94.

86. - Structure of genetic algebras, Amer. J. Math. vol. 71 (1949) pp. 121135.

87. - The Wedderburn principal theorem for alternative algebras, Bull. Amer. Math. Soc. vol. 55 (1949) pp. 604-614.

88. —-, Inner derivations of non-associative algebras, Bull. Amer. Math. Soc. vol. 55 (1949) pp. 769-776.

89. - A theorem on the derivations of Jordan algebras, Proc. Amer. Math. Soc. vol. 2 (1951) pp. 290-294.

90. — Representations of alternative algebras, Trans. Amer. Math. Soc. vol. 72 (1952) pp. 1-17. 
91. - The Casimir operation for alternative algebras, Proc. Amer. Math. Soc. vol. 73 (1953) pp. 444-451.

92. —, A generalization of a theorem of Albert, Proc. Amer. Math. Soc. vol. 73 (1953) pp. 452-455.

93. - On the algebras formed by the Cayley-Dickson process, Amer. J. Math. vol. 76 (1954) pp. 435-446.

94. - Noncommutative Jordan algebras of characteristic 0, Proc. Amer. Math. Soc. vol. 6 (1955) pp. 472-475.

95. L. A. Skornyakov, Natural domains of Veblen-Wedderburn projective planes, Izvestiya Akad. Nauk SSSR vol. 13 (1949) pp. 447-472 (Russian). Amer. Math. Soc. Translation no. 58.

96. —-, Alternative fields, Ukrain. Mat. Zurnal vol. 2 (1950) pp. 70-85 (Russian).

97. — , Right-alternative fields, Izvestiya Akad. Nauk SSSR Ser. Mat. vol. 15 (1951) pp. 177-184 (Russian).

98. — Projective planes, Uspehi Matematičeskih Nauk (N.S.) vol. 6 (1951) pp. 112-154 (Russian). Amer. Math. Soc. Translation no. 99.

99. M. F. Smiley, The radical of an alternative ring, Ann. of Math. vol. 49 (1948) pp. 702-709.

100. - Application of a radical of Brown and McCoy to non-associative rings, Amer. J. Math. vol. 72 (1950) pp. 93-100.

101. - On the ideals and automorphisms of non-associative rings, Proc. Amer. Math. Soc. vol. 2 (1951) pp. 138-143.

102. - Some questions concerning alternative rings, Bull. Amer. Math. Soc. vol. 57 (1951) pp. 36-43.

103. E. Stiefel, On Cauchy-Riemann equations in higher dimensions, Journal of Research of the National Bureau of Standards vol. 48 (1952) pp. 395-398.

104. O. Taussky, An algebraic property of Laplace's differential equation, Quart. J. Math. vol. 10 (1939) pp. 99-103.

105. M. L. Tomber, Lie algebras of type F, Proc. Amer. Math. Soc. vol. 4 (1953) pp. 759-768.

106. J. von Neumann, On an algebraic generalization of the quantum mechanical formalism, Mat. Sbornik vol. 1 (1936) pp. 415-482.

107. F. B. Wright, Absolute valued algebras, Proc. Nat. Acad. Sci. U.S.A. vol. 39 (1953) pp. 330-332.

108. J. L. Zemmer, Jr., On the subalgebras of finite division algebras, Canadian Journal of Mathematics vol. 4 (1952) pp. 491-503.

109. M. Zorn, Theorie der alternativen Ringe, Abh. Math. Sem. Hamburgischen Univ. vol. 8 (1930) pp. 123-147.

110. - Alternativkörper und quadratische systeme, Abh. Math. Sem. Hamburgischen Univ. vol. 9 (1933) pp. 395-402.

111. - The automorphisms of Cayley's non-associative algebra, Proc. Nat. Acad. Sci. U.S.A. vol. 21 (1935) pp. 355-358.

112. - Alternative rings and related questions I: Existence of the radical, Ann. of Math. vol. 42 (1941) pp. 676-686.

\section{University of Connecticut}

